\title{
PREDICTION OF GRAFT SURVIVAL USING ARTIFICIAL NEURAL NETWORK (ANN), AND BAYESIAN BELIEF NETWORK (BBN): A COMPARATIVE STUDY
}

\section{---Graft Survival Prediction using Machine Learning: A Comparative Study}

C. Theophilus Dhyankumarl, C. Joe Arun', M. Rajmohan²

1. Loyola Institute of Business Administration (LIBA), Chennai, Tamilnadu, India.

2. College of Engineering, Guindy, Anna University, Chennai, Tamilnadu, India

Correspondence: theophilus.c@liba.edu

\section{ABSTRACT}

\section{OBJECTIVE:}

The aim of this study was to predict graft survival using machine learning prediction techniques and the involved decision making.

\section{DESIGN:}

Prediction of graft survival post-transplant using machine learning algorithms like Artificial Neural Network (ANN) (Single and Multi-layer networks), and Bayesian Belief Network (BBN).

\section{SETTING:}

Recipient and donor with characteristics of age, sex and Glomerular Filtration Rate (GFR) and the follow-up of probability of survival one year after transplantation $(n=40)$.

\section{MAIN OUTCOME MEASURES:}

The Data include simulation from donor, recipient characteristics of single centre with factors age, sex, GFR and probability of survival collected particularly with the follow-up after the first year of transplant.

\section{RESULTS:}

The ANN and BBN were modelled in Python. The probability of survival post-transplant is predicted, and accuracy measured using Root Mean Square Error (RMSE). The results for the methods were compared and efficacy and ease of use are discussed.

\section{CONCLUSION:}

The decision making in the organ transplantation involving the patients and doctors consists of mainly involve improving the graft survival and hence prediction becomes important. The developed models can be used to predict the transplant and aid as decision support system for decision regarding matching and allocation.

\section{KEYWORDS}

Organ Transplantation, Graft survival prediction, Artificial Neural Network (ANN), Bayesian Belief Network (BBN). 
INTRODUCTION

Organ Transplantation is to place the functioning organ obtained from a live/ dead donor or grown in a lab and onto the patient whose organ function is either deteriorating or has completely stopped functioning and needs assistance from an external source. One of the reasons for long term graft survival after transplantation is to go for best match through blood group compatibility. There are reasons like age, medical condition and compliance degree of the patients that also contribute to the graft survival. The advancement of medical technology has provided a better solution for reducing the rejection of the graft, in the form of treatment with immunosuppressant drugs.

The literature on prediction of graft survival is available involving factors and characteristics of recipients and donors. The studies most relevant to our study especially with respect to the prediction techniques, Artificial Neural Network (ANN) and Bayesian Belief Networks (BBN) are explained briefly in this section.

Hariharan et al. [1] considered only pre-transplant data like sex, age and race which are demographic data and medical characteristics like HLA and diabetes of recipient and type and age of donor. Baskin-Bey et al. [2] considered type of donor and its effect on graft survival. Both the studies used the US renal data base for the analysis. Machiniki et al. [3] took notice on the effect of age and medical factors like hypertension, cause of the chronic kidney disease, life style factors like usage of tobacco, duration of the period of dialysis before transplant. Bertram et al. [4] considered around 38 recipient and donor characteristics most of them were related to posttransplant characteristics.

The work of Gumber et al. [5] was in deceased donor organ transplantation especially for a 4-year survival prediction and he found $77.5 \%$ patient survival and $89.3 \%$ graft survival. Sharma et al. [6] studied the delayed graft function considering factors like age and hypertension of the recipient. Osofisan et al. [7] predicted failure of kidney based on the traces of creatinine, chloride, potassium, urea and uric acid in urine. Brier et al. [8] used ANN to predict delayed graft function like sex, age, weight, height, body surface area and race for recipient and gender, age, HLA and cold ischemia time for donor. Shadabi and Sharma [9] compared the performance of their developed
ANN model and Logistic regression model for predicting the survival of graft at the end of the second year after transplantation.

Topuz et al. [10] performed analysis of retrospective database, used sensitivity analyses and information fusion to evaluate and combine features from several machine learning approaches (Support vector machine (SVM), ANN and Bootstrap Forest). They used best performing sets in Bayesian belief network (BBN) algorithm to identify nonlinear relationships and the interactions between explanatory factors and risk levels for kidney graft survival. An intelligent system for medical decision making for organ transplantation was initiated by Perris and Labib [11] by introducing two fuzzy logic models for improving the then prioritization system used in the UK and abroad. The first model converts an element of the current kidney transplant prioritization system used in the UK into fuzzy logic. The result is an improvement to the current system and a demonstration of the usage of fuzzy logic as an effective decision-making tool.

From the literature it is evident that ANN is used for prediction of delayed graft function and graft survival after 2 years, in a similar way we have developed a feed forward perceptron single and multi-layered model for a prediction of 1 st year post-transplant for Indian scenario. Similarly, Bayesian Belief Network (BBN) was developed for the same data

The prediction of graft survival has been extensively studied but the proposed model compares the prediction using Artificial Neural Network andBayesian Belief Network. Section 3 deals with the methodology developed for the study. The results and discussion are explained in Section 4 followed by the conclusion in Section 5 .

\section{METHODOLOGY}

The Prediction of graft survival using Artificial Neural Network (ANN) in single layer and multiple layer, Bayesian Belief Network (BNN) and Fuzzy logic are explained with respect to the problem in this section.

\section{PREDICTION OF GRAFT SURVIVAL USING ARTIFICIAL NEURAL NETWORK (ANN)}

The Artificial Neural Network (ANN) tries to imitate the biological neuron of the brain's pattern of learning. It basically consists of input, processing and output elements. There may be several layers in between the input and 
output layer too either open or hidden and it can be categorized based on that into single layer and multi layered network models.

In this work both single layered and multi layered feed forward back propagation perceptron neural network model has been developed for prediction of graft survival taking the criteria namely age and sex of both donor and recipient, and Glomerular Filtration Rate (GFR) calculated from the serum creatinine level and the probability of survival post-transplant of the recipient into account. The number of hidden layers and the units in each layer was determined based on the minimum sum of squares error. The same logic is used for modelling the ANN separately for the single as well as multi layered network.

\section{SINGLE-LAYER PERCEPTRON (SLP)}

A single-layer perceptron network consists of one or more artificial neurons in parallel. The neurons may be of the same type we've seen in the Artificial Neuron Applet. Each neuron in the layer provides one network output, and is usually connected to all of the external inputs. The network architecture and the pseudo code of the single layer perceptron network for the problem are also given.

\section{FIGURE 1: SINGLE LAYERED PERCEPTRON ARCHITECTURE}

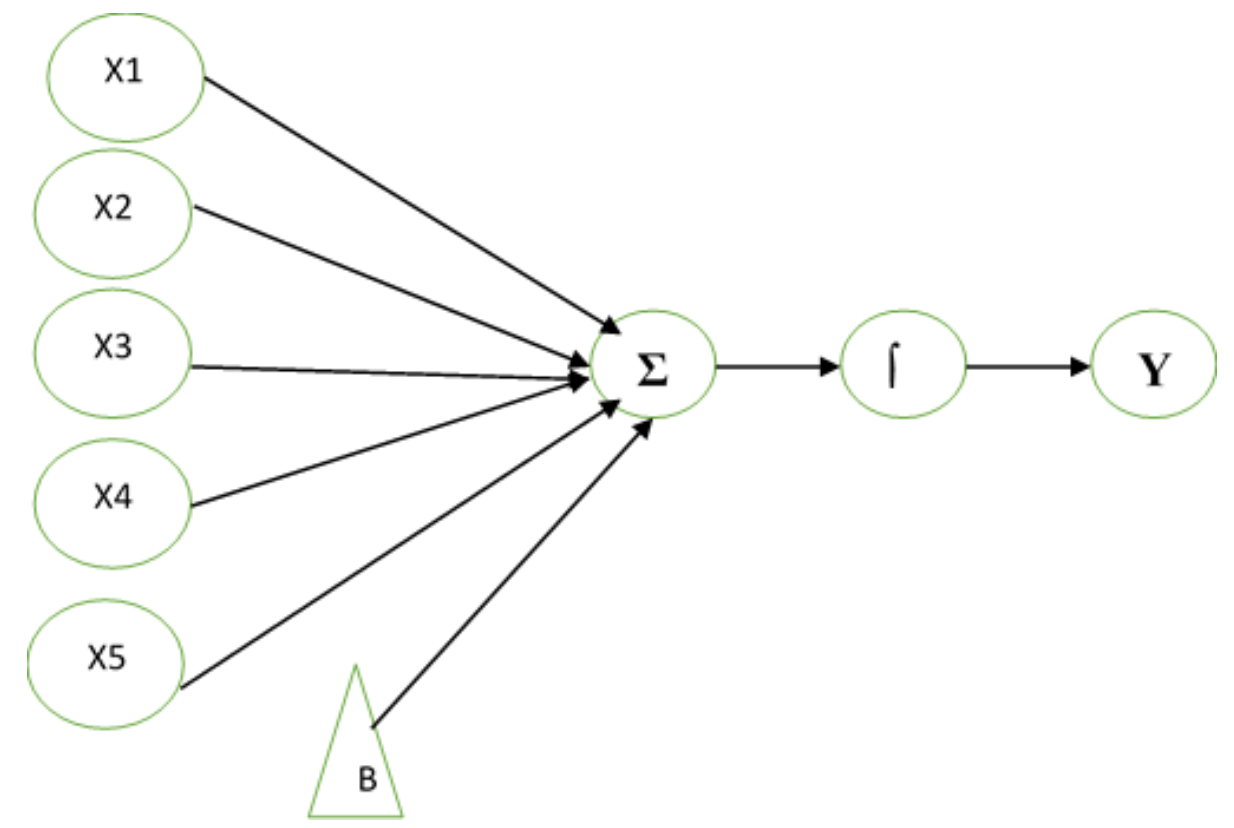

The input variable, output variable, activation functions and their abbreviations are explained below:

$\mathrm{X} 1$

\begin{tabular}{lll} 
& - & \multicolumn{2}{l}{ Receptor Age } \\
X2 & - & Donor Age \\
X3 & - & Receptor Sex \\
X4 & - & Donor Sex \\
X5 & - & Glomerulus Filtration Rate \\
B & - & Bias \\
$\Sigma$ & - & Summation Function \\
& & Sigmoid Function
\end{tabular}

$\mathrm{F}(\mathrm{z})=1 /\left(1+e^{-\mathrm{z}}\right)$

Y $\quad$ - $\quad$ Probability of Graft Survival

\section{MULTI-LAYER PERCEPTRON (MLP)}

A Multi-Layer Perceptron is a larger network of simple neurons called perceptrons. The perceptron calculates a single output from multiple inputs by summing up a linear combination of its input and weights and then applying it upon the output through some nonlinear activation function.

\section{MATHEMATICALLY THIS CAN BE WRITTEN AS}

$y=\varphi\left(\sum_{i=1}^{N} w * x+b\right)$

Where,

$\begin{array}{lll}w & - & \text { Vector of weights, } \\ x & - & \text { Vector of inputs, } \\ b & - & \text { Bias, } \\ \varphi & - & \text { Activation function. }\end{array}$


A single perceptron has limited mapping ability, and hence not robust. A basic multilayer perceptron network consists of a set of nodes in the input layer, one or more hidden layers of computation nodes, and an output layer which may again be single or a number of nodes. The input signal is cascaded through the network one layer at a time.

\section{PREDICTION OF GRAFT SURVIVAL USING BAYESIAN BELIEF NETWORKS (BBN)}

Bayesian models are probabilistic graphical models used to build prediction models from the data as well as the opinions from the experts. The naive Bayes classification technique is used for classifying the recipients of the organs post-transplant of their survival after the first year of transplant. It is a supervised technique and is a simple model assuming independence between predictor

variables and the target for learning the structure. According to Bayes rule the probability is calculated for each attribute variable using the following formula.

$$
\text { Posterior }=\frac{\text { prior*likelihood }}{\text { evidence }}
$$

The training data contains the donor age, recipient age, GFR all are assumed as continuous variables following Gaussian probability distribution. The block diagram consisting of the input variables, Bayesian equation and output in terms of the survival probability is shown in Figure 2.

FIGURE 2: BLOCK DIAGRAM OF BAYESIAN MODEL FOR PREDICTING PROBABILITY OF GRAFT SURVIVAL

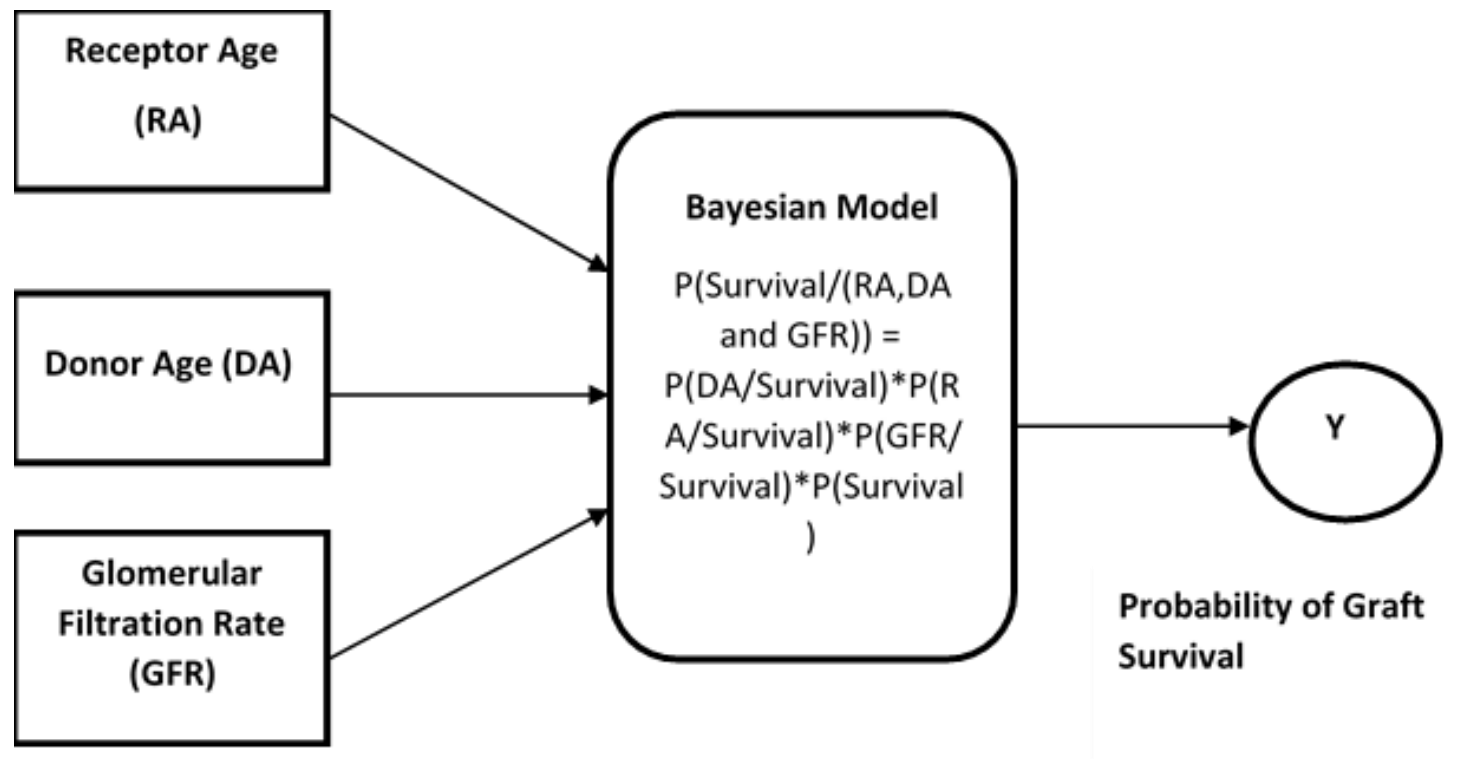

FIGURE 3: CONVERGENCE PLOT FOR SINGLE-LAYERED ANN MODEL

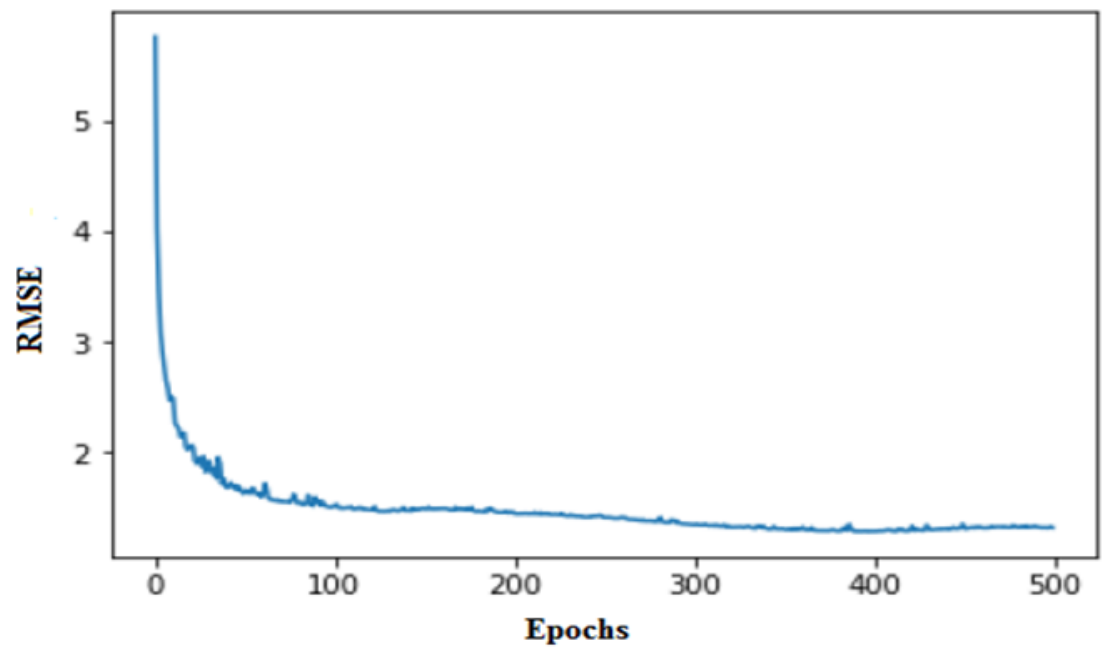


The data used for prediction is generated for 40 recipients and 40 donors containing the factors like sex, age, Glomerular Filtration Rate (GFR) and probability of survival and is available. In the analysis of the graft survival, time is an important factor that needs to be taken into account. The function of the graft at the end of the 1st year given the characteristics of the recipient that is matched with the donor is considered in this work.

\section{GRAFT SURVIVAL USING ARTIFICIAL NEURAL NETWORK (ANN)}

The ANN was modelled in Python and the data of 40 recipients and donors is used for training. The number of hidden layers and the units in each layer was determined based on the minimum sum of squares error.

The code was run in Python and the results obtained were plotted in the form of graph in Figure 3 with number of epochs in $x$-axis and root mean squared error (RMSE) in the $y$-axis. The plot assesses convergence and stops the analysis when the solution is no longer changing. It can be seen that the error value decreases gradually as the number of iterations increase to a maximum of 500 . However, single-layer perceptron networks cannot learn everything that is computationally incomplete. In order to overcome this disadvantage, the multilayer perceptron (MLP) has been developed.
The code was run in Python and results obtained are shown in graph. From the convergence plot in Figure 4, the Error vs. Epochs curve is steeply decreasing in the second iteration itself thus ensuring superior accuracy in comparison to single layer perceptron.

\section{GRAFT SURVIVAL USING BAYESIAN BELIEF NETWORK (BBN)}

The data obtained consists of the donor age, receptor age, GFR and probability of graft survival. Age is represented as continuous values by finding the mean and the standard deviation of the available data and also for the GFR. All of these are prepared based on the sample data created from the history of data and is shown separately for recipients and donors in Tables 1 and 2 .

The data taken as continuous values for receptor age, donor age and GFR (Table 4) was used to predict the probability of graft survival based on the Bayesian Belief Network (BBN) and was executed using MATLAB.

The continuous values are obtained through normal probability function and the probability is calculated. Though outliers are obtained from the developed model it is only because of the randomness in the model, thus proving that it is neither over fit nor under fit.

A sample data was assumed and the results are compared with ANN and BBN in Table 4.

FIGURE 4: CONVERGENCE PLOT FOR MULTI-LAYERED ANN MODEL

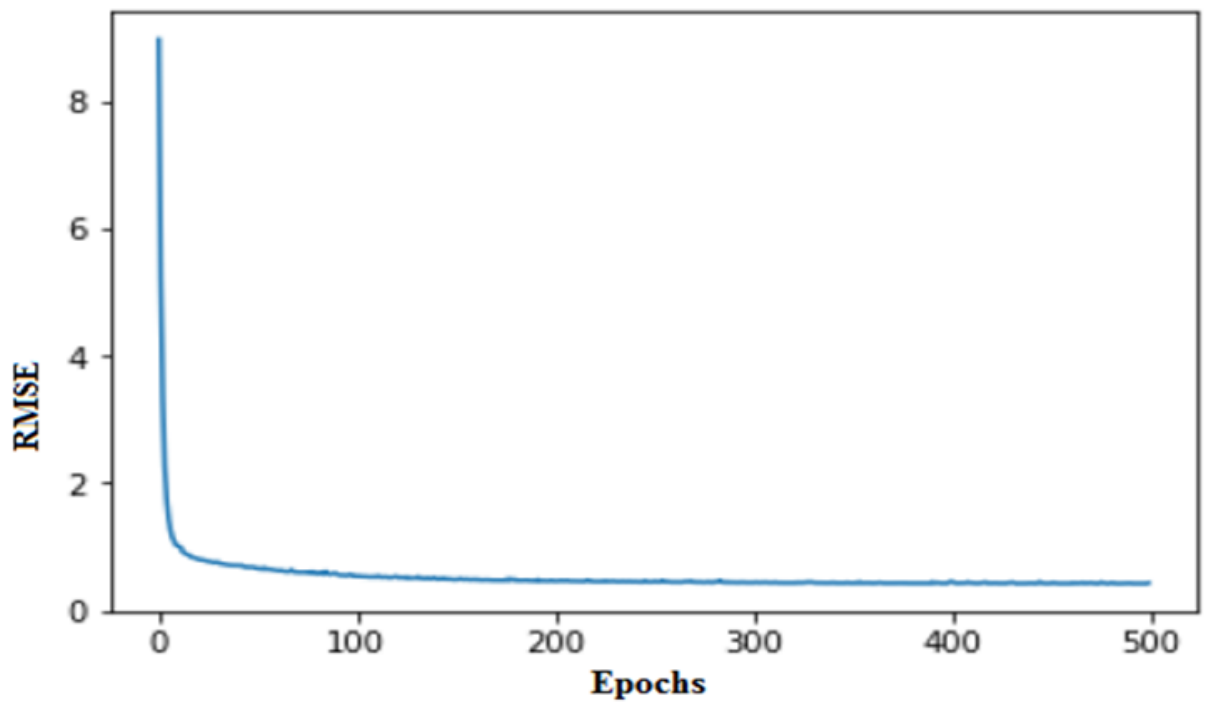


TABLE 1: RECEPTOR DATA

\begin{tabular}{|l|l|l|l|l|}
\hline SL. NO. & DESCRIPTION & YES & NO & $\begin{array}{l}\text { PROBABILITY OF } \\
\text { M/F SURVIVES }\end{array}$ \\
\hline 1 & Male & 22 & 17 & 0.494974874 \\
\hline 2 & Female & 28 & 13 & 0.505025126 \\
\hline 3 & All & 50 & 30 & - \\
\hline 4 & Prob(Yes/Male) & 0.44 & 0.566667 & - \\
\hline 5 & Prob(Yes/Female) & 0.56 & 0.433333 & - \\
\hline
\end{tabular}

TABLE 2: DONOR DATA

\begin{tabular}{|l|l|l|l|l|}
\hline $\begin{array}{l}\text { SL. } \\
\text { NO. }\end{array}$ & DESCRIPTION & YES & NO & $\begin{array}{l}\text { PROBABILITY } \\
\text { M/F }\end{array}$ \\
\hline 1 & Male & 24 & 12 & 0.454774 \\
\hline 2 & Female & 26 & 18 & 0.545226 \\
\hline 3 & All & 50 & 30 & - \\
\hline 4 & Prob(Yes/Male) & 0.48 & 0.4 & - \\
\hline 5 & Prob(Yes/Female) & 0.52 & 0.6 & - \\
\hline
\end{tabular}

TABLE 3: INPUT AND OUTPUT VALUES FOR GRAFT SURVIVAL

\begin{tabular}{|l|l|l|l|l|}
\hline SL. NO. & $\begin{array}{l}\text { RECEPTOR } \\
\text { AGE (RA) }\end{array}$ & $\begin{array}{l}\text { DONOR } \\
\text { AGE (DA) }\end{array}$ & GFR & $\begin{array}{l}\text { PROBABILITY } \\
\text { OF GRAFT SURVIVAL (Y) }\end{array}$ \\
\hline 1 & 0.23 & 0.45 & 0.89 & 0.85 \\
\hline 2 & 0.31 & 0.47 & 0.648 & 0.70 \\
\hline 3 & 0.85 & 0.73 & 0.24 & 0.38 \\
\hline 4 & 0.58 & 0.49 & 0.66 & 0.57 \\
\hline 5 & 0.65 & 0.36 & 0.85 & 0.45 \\
\hline
\end{tabular}

TABLE 4: COMPARISON OF ANN AND BBN MODELS

\begin{tabular}{|l|l|l|}
\hline SL. NO. & MODEL & $\begin{array}{l}\text { PROBABILITY OF GRAFT } \\
\text { SURVIVAL }\end{array}$ \\
\hline 1 & Single-layer perceptron & 0.525 \\
\hline 2 & Multi-layer perceptron & 0.609 \\
\hline 3 & Bayesian model & 0.648 \\
\hline
\end{tabular}


The convergence plot for both the single and multi-layered network model were developed. Thus multilayer perceptron neural network with the above architecture enables us to predict the probability of the graft survival with reduced error. The convergence of multi layered network happens to be more real compared to the single layered network. The comparison is also done between ANN and BBN model.

\section{CONCLUSION}

The prediction of graft survival post-transplant is an important area in making decision on the allocation of organs between the recipients and donors based on the data on follow up after transplantation. In this study prediction of the graft survival in organ transplantation using the techniques like Artificial Neural Network (ANN) (Single layer and multi-layer perceptron), and Bayesian Belief Network (BBN) and comparison for a small sample of data has been carried out. The accuracy of prediction of the methods is measured in terms of the probability of survival of graft value provided. Above all the application of the prediction techniques by the decision makers in making the decision of allocation in future is very important, depending on the graft survival probability of the allocated organ to the recipient. Ultimately the final decision is made by the doctors, who are the experts, as the solution aids them in making the decisions of allocation of the organ to the best matching patient.

\section{References}

1. Hariharan S, Johnson CP, Bresnahan BA, Taranto SE, McIntosh MJ, Stablein D. Improved graft survival after renal transplantation in the United States, 1988 to 1996. N Engl J Med, 2000 Mar;342(9):605-612.

2. Baskin-Bey ES, Kremers W, Nyberg SL. A recipient risk score for deceased donor renal allocation. Am J Kidney Dis. 2007;49:284-93.

3. Machnicki G, Pinsky B, Takemoto S, Balshaw R, Salvalaggio PR, Buchanan PM, Irish W, Bunnapradist S, Lentine KL, Burroughs TE, Brennan DC, Schnitzler MA. Predictive ability of pretransplant comorbidities to predict long-term graft loss and death. Am J Transplant 2009 Mar;9(3):494-505.
4. Bertram LK, Ajay KI, Jon JS, Melissa AS, Yipeng, Eric DW. A simple tool to predict outcomes after kidney transplant. Am J Kidney Dis. 2010 Nov;56:947-960.

5. Gumber MR, Gopalani KR, Shah PR, Patel HV. Study on deceased donor organ transplantation, Indian J Nephrol $2011 ; 21$ (3):182-186.

6. Sharma AK, Tolani SL, Rathi GL, Sharma P, Gupta H, Gupta R. Evaluation of factors causing delayed graft function in live related donor renal transplantation. Saudi J Kidney Dis Transpl 2010 Mar;21 (2):242-5.

7. Osofisan AO, Adeyemo OO, Sawyerr BA. Prediction of Kidney Failure Using Artificial Neural Networks. Eur J Sci Res $2011 ; 61(4): 487-492$.

8. Brier MV, Ray PC, Klein B. Prediction of delayed renal dialysis allograft function using an artificial neural network. Nephrol Dial Transplant 2003; 18(12):2655-9.

9. Shadabi F, Sharma D. Comparison of artificial neural networks with logistic regression in prediction of kidney transplant outcomes. International Conference on IEEE, p 543-7.

10. Topuz K, Zengul FD, Dag A, Almehmi A, Yildirim MB. Predicting graft survival among kidney transplant recipients: A Bayesian decision support model. Decis Support Syst 2018;106:97-109.

11. Perris T, Labib AW. An intelligent system for prioritisation of organ transplant patient waiting lists using fuzzy logic. J Oper Res Soc 2004 Feb. Special issue: Intelligent management systems in operations;55(4):103-115. 